\title{
MEAN CONVERGENCE OF GRÜNWALD INTERPOLATION OPERATORS
}

\author{
ZHIXIONG CHEN
}

Received 5 November 2002

\begin{abstract}
We investigate weighted $L^{p}$ mean convergence of Grünwald interpolation operators based on the zeros of orthogonal polynomials with respect to a general weight and generalized Jacobi weights. We give necessary and sufficient conditions for such convergence for all continuous functions.
\end{abstract}

2000 Mathematics Subject Classification: 41A35.

1. Introduction. In this paper, we study weighted $L^{p}(0<p<\infty)$ mean convergence of Grünwald interpolation operators which was introduced in [3]. We first consider the weighted convergence for the Grünwald interpolation operator when a general weight is used. We also consider in particular the weighted $L^{p}$ convergence for the Grünwald interpolation operator when a generalized $J a$ cobi weight is used. Necessary and sufficient conditions for such convergence for a continuous function are presented. In Section 1, we briefly introduce the Hermite-Fejér interpolation and Grünwald interpolation. In Section 2, we review some known results that are closely related to the main results of this paper and will be used in our proof later. We also establish several preliminary results. In Section 3, we state and prove the main results of this paper.

We first introduce the Hermite-Fejér interpolation polynomials and the Grünwald interpolation operator. Let $w$ be a weight function of interval $[-1,1]$ and $\left\{P_{n}(w, x)\right\}$ the orthonormal polynomials on $[-1,1]$ with respect to $w$. Assume that we are given a system $X$ of nodes

$$
X:\left(x_{0} \equiv x_{0, n} \equiv\right) 1 \geq x_{1 n}>x_{2 n}>\cdots>x_{n n} \geq-1\left(\equiv x_{n+1, n} \equiv x_{n+1}\right),
$$

The Hermite-Fejér interpolation polynomials of $f \in C[-1,1]$ at $X$ are defined by

$$
H_{n}(X, f, x):=\sum_{k=1}^{n} f\left(x_{k}\right) v_{k}(x) l_{k}^{2}(x)
$$


where

$$
\begin{aligned}
& v_{k}(x)=1-\frac{w_{n}^{\prime \prime}\left(x_{k n}\right)}{w_{n}^{\prime}\left(x_{k n}\right)}\left(x-x_{k n}\right), \quad k=1,2, \ldots, n, \\
& l_{k n}(x)=\frac{w_{n}(x)}{\left(x-x_{k n}\right) w_{n}^{\prime}\left(x_{k n}\right)}, \quad k=1,2, \ldots, n, n=1,2, \ldots, \\
& w_{n}(x)=\left(x-x_{1 n}\right)\left(x-x_{2 n}\right) \cdots\left(x-x_{n n}\right), \quad n=1,2, \ldots
\end{aligned}
$$

We use $l_{k n}(w, x)$ and $H_{n}(w, f, x)$ to denote $l_{k n}(X, x)$ and $H_{n}(X, f, x)$, respectively, when the set $X$ is chosen to be the zeros of the orthogonal polynomial $P_{n}(w, x)$. For simplicity, we substitute $x_{k}$ for $x_{k n}$. The Christoffel function is defined by

$$
\lambda_{n}(w, x):=\left[\sum_{k=0}^{n-1} P_{k}^{2}(w, x)\right]^{-1}=\left[\sum_{k=1}^{n} \frac{l_{k n}^{2}(w, x)}{\lambda_{k n}}\right]^{-1}, \quad n=1,2, \ldots
$$

The numbers $\lambda_{k n}(w)$, defined by

$$
\lambda_{k n}=\lambda_{n}\left(w, x_{k n}\right)=\int_{-1}^{1} l_{k n}^{2}(w, x) w(x) d x, \quad k=1,2, \ldots, n, n=1,2, \ldots,
$$

are called the Cotes numbers. It is well known (see [2, page 113], [9]) that

$$
\begin{aligned}
H_{n}(w, f, x)= & \sum_{k=1}^{n} f\left(x_{k n}\right)\left[1+\frac{\lambda_{n}^{\prime}\left(w, x_{k n}\right)}{\lambda_{k n}}\left(x-x_{k n}\right)\right] l_{k n}^{2}(x) \\
= & \sum_{k=1}^{n} f\left(x_{k n}\right)\left[1-\frac{P_{n}^{\prime \prime}\left(w, x_{k n}\right)}{P_{n}^{\prime}\left(w, x_{k n}\right)}\left(x-x_{k n}\right)\right] l_{k n}^{2}(x), \\
& -\frac{P_{n}^{\prime \prime}\left(w, x_{k n}\right)}{P_{n}^{\prime}\left(w, x_{k n}\right)}=\frac{\lambda_{n}^{\prime}\left(w, x_{k n}\right)}{\lambda_{k n}} .
\end{aligned}
$$

If $P$ is a polynomial of degree at most $2 n-1$, then

$$
P(x)=H_{n}(X, P, x)+\sum_{k=1}^{n} P^{\prime}\left(x_{k n}\right)\left(x-x_{k n}\right) l_{k n}^{2}(x) .
$$

The Grünwald interpolation polynomial of $f \in C[-1,1]$ at $X$ is defined by

$$
G_{n}(f, x):=G_{n}(X, f, x):=\sum_{k=1}^{n} f\left(x_{k n}\right) l_{k n}^{2}(x), \quad k=1,2, \ldots, n,-1 \leq x \leq 1 .
$$

If $u \geq 0$ and $0<p<\infty$, then $f \in L_{u}^{p}$ provided that $\|f\|_{u, p}<\infty$, where

$$
\|f\|_{u, p}:=\left[\int_{-1}^{1}|f(t)|^{p} u(t) d t\right]^{1 / p}
$$


Naturally, when $0<p<1,\|\cdot\|_{u, p}$ is not a norm. The function $u(x)$ is called a Jacobi weight function if $u(x)$ can be written as $u^{(a, b)}(x)=(1-x)^{a}(1+b)^{b}$, $-1 \leq x \leq 1, a, b>-1$, and $u(x)=0$ if $|x|>1$. (The function $u(x)$ is the simple form of $u^{(a, b)}(x)$.) The function $u(x)$ is a generalized Jacobi weight function $(u \in \mathrm{GJ})$ if $u \in L^{1}$ and $u$ can be written in the form $u(x)=g(x)(1-x)^{a}(1+$ $x)^{b}$, where $g>0$ and $g^{ \pm 1} \in L^{\infty}$.

The uniform convergence of the corresponding Grünwald interpolation was investigated by several authors [3, 6], and $L^{p}$ convergence for such interpolation was studied in [5] with $p=1$ only. Here, for convenience, we state the theorem which was proved in [5].

If $f \in C[-1,1],\left\{x_{k}\right\}$ are zeros of Jacobi function $J_{n}^{a, b}(x)(-1<a, b<1)$, then

$$
\lim _{n \rightarrow \infty} \int_{-1}^{1}\left|G_{n}(f, x)-f(x)\right| d x=0
$$

2. Auxiliary propositions. In this section, we obtain some preliminary results. First, we need the following notations. Here and later the symbols const and $C$ denote some positive constants, not necessarily having the same values in different formulas. If $A$ and $B$ are two expressions depending on some variables and indices, then $A \sim B \Leftrightarrow\left|A B^{-1}\right| \leq$ const and $\left|A^{-1} B\right| \leq$ const. Let $w$ be a generalized Jacobi weight function ( $w \in \mathrm{GJ}$ ). In the following, we summarize some results from [8] that will be useful for our development in this paper. Assume that

$$
\begin{gathered}
x_{k n}=x_{k n}(w)=\cos \theta_{k n}, \quad x_{0 n}=\cos \theta_{0 n}=1, \\
x_{n+1, n}=\cos \theta_{n+1, n}=-1, \quad 0 \leq \theta_{k n} \leq \pi, \\
\left|x-x_{j}\right|=\min _{0 \leq k \leq n+1}\left|x-x_{k}\right|, \quad 0 \leq j \leq n+1 .
\end{gathered}
$$

Then we have

$$
\begin{gathered}
\theta_{k+1, n}-\theta_{k n} \sim \frac{1}{n}, \quad k=0,1, \ldots, n, \\
\left|x-x_{k n}\right| \sim \frac{|k-j| \min \{k+j, 2 n+2-k-j\}}{n^{2}}, \quad 1 \leq k \leq n, k \neq j .
\end{gathered}
$$

We also have the estimates for the orthonormal polynomial $P_{n}(w, x)$ :

$$
\begin{aligned}
& P_{n}^{\prime}\left(w, x_{k n}\right) \sim n w\left(x_{k n}\right)^{-1 / 2}\left(1-x_{k n}^{2}\right)^{-3 / 4}, \\
& \left|P_{n}(w, x)\right| \leq \mathrm{const} \begin{cases}{\left[w(x)\left(1-x^{2}\right)^{1 / 2}\right]^{-1 / 2},} & |x| \leq 1-n^{-2}, \\
n^{1 / 2} w\left(1-n^{-2}\right)^{-1 / 2}, & 1-n^{-2} \leq x \leq 1, \\
n^{1 / 2} w\left(-1+n^{-2}\right)^{-1 / 2}, & -1 \leq x \leq-1+n^{-2},\end{cases}
\end{aligned}
$$


uniformly for $n \geq 2$,

$$
\left|P_{n}(w, x)\right| \sim \begin{cases}n\left|x-x_{j n}\right|\left[w(x)\left(1-x^{2}\right)^{3 / 2}\right]^{-1 / 2}, & -1+x_{n 1} \leq 2 x \leq 1+x_{1 n}, \\ n^{1 / 2} w\left(1-n^{-2}\right)^{-1 / 2}, & 1+x_{1 n} \leq 2 x \leq 2, \\ n^{1 / 2} w\left(-1+n^{-2}\right)^{-1 / 2}, & -2 \leq 2 x \leq 1+x_{n n},\end{cases}
$$

uniformly for $n \geq 2$.

For the Cotes numbers, there hold

$$
\lambda_{k n}(w) \sim \frac{1}{n} w\left(x_{k n}\right)\left(1-x_{k n}^{2}\right)^{1 / 2},
$$

uniformly for $1 \leq k \leq n, n \in \mathbb{N}$,

$$
\left|\lambda_{n}^{\prime}\left(w, x_{k n}\right)\right| \leq \text { const } \frac{1}{n} w\left(x_{k n}\right)\left(1-x_{k n}^{2}\right)^{-1 / 2} .
$$

The following inequality is also needed:

$$
(|a|+|b|)^{p} \leq 2^{p}\left(|a|^{p}+|b|^{p}\right) .
$$

LEMMA 2.1. Let $f(x) \in C[a, b]$ and let $L_{n}$ be a linear positive operator. Then the following statements are equivalent:

(1) $\left\|L_{n} f-f\right\|_{u, p} \rightarrow 0$, for every $f \in C[a, b]$;

(2) $\left\|L_{n} f-f\right\|_{u, p} \rightarrow 0, f(x)=1, x, x^{2}$;

(3) $\left\|L_{n} 1-1\right\|_{u, p} \rightarrow 0$ and $\left\|\left(L_{n} \phi_{t}\right)(t)\right\|_{p} \rightarrow 0$, where $\phi_{t}(x) \equiv(t-x)^{2}$.

Proof. By using the proposition in [4, page 153] and the theorem on monotone operators in [1, page 67], we know that this lemma holds.

LEMмA 2.2 [8, Theorem 1, page 46]. Let $w$ be a general weight function. Then

$$
\lim _{n \rightarrow \infty} H_{n}(w, P)=P
$$

in $L_{w}^{1}$ for every polynomial $P$.

LEMMA 2.3 [7, Theorem 6.3.14, page 113]. For every $0<p<\infty$ and every Jacobi weight function $u$, there exists a constant $\sigma=\sigma(p, u)>0$ such that, for every polynomial $P$ of degree less than $2 n$,

$$
\int_{-1}^{1}|P(x)|^{p} u(x) d x \leq 2 \int_{-1+\sigma n^{-2}}^{1-\sigma n^{-2}}|P(x)|^{p} u(x) d x
$$


LEMMA 2.4 [8, Theorem 4, page 53]. Let $w \in \mathrm{GJ}$, let $u, v$ be two Jacobi weight functions, and let $p>0$. Then

$$
\lim _{n \rightarrow \infty} H_{n}(w, R)=R
$$

in $L_{u}^{p}$ for every polynomial $R$ satisfying the condition $|R(x)| \leq$ const $v(x),-1 \leq$ $x \leq 1$ if and only if $w^{-1} \in L_{u}^{p}$, in particular, $p$ is independent of $v$.

LEMMA 2.5. Let $w \in \mathrm{GJ}$ and $0<\sigma<1$. Then there hold

$$
\begin{aligned}
& \sum_{k=1}^{n} \frac{\left|\lambda_{n}^{\prime}\left(w, x_{k}\right)\right|}{\lambda_{k}(w)}\left|x-x_{k}\right| l_{k}^{2}(x) \\
& \quad \leq \text { const }\left[\frac{1}{n^{2 \alpha+2}}+\frac{1}{n^{2 \beta+2}}+\frac{\ln n}{n}\right] \frac{1}{w(x)\left(1-x^{2}\right)}
\end{aligned}
$$

uniformly for $n \geq 2$ and $|x| \leq 1-\sigma n^{-2}$ and

$$
\begin{aligned}
& \sum_{k=1}^{n} \frac{\left|\lambda_{n}^{\prime}\left(w, x_{k}\right)\right|}{\lambda_{k}(w)}\left|x-x_{k}\right| l_{k}^{2}(x) \\
& \quad \leq \text { const }\left[\frac{1}{n^{2 \alpha+1}}+\frac{1}{n^{2 \beta+1}}+\frac{\ln n}{n}\right] \frac{1}{w(x)\left(1-x^{2}\right)^{1 / 2}}
\end{aligned}
$$

uniformly for $n \geq 2$ and $|x| \leq 1-\sigma n^{-2}$.

Proof. Let $0 \leq x \leq 1-\sigma n^{-2}$. Then $j \leq n / 2$.

First, by (2.6) and (2.7), we get

$$
\frac{\left|\lambda_{n}^{\prime}\left(x_{k}\right)\right|}{\lambda_{k}} \leq C \frac{(1 / n) w\left(x_{k}\right)\left(1-x_{k}^{2}\right)^{-1 / 2}}{(1 / n) w\left(x_{k}\right)\left(1-x_{k}^{2}\right)^{1 / 2}} \leq C \frac{1}{1-x_{k}^{2}},
$$

which implies that

$$
\frac{\left|\lambda_{n}^{\prime}\left(x_{j}\right)\right|}{\lambda_{j}}\left|x-x_{j}\right| l_{j}^{2}(x) \leq C \frac{1}{1-x_{j}^{2}} \frac{P_{n}^{2}(x)}{P_{n}^{\prime}\left(x_{j}\right)^{2}\left|x-x_{j}\right|} .
$$

Next, using (2.4) and (2.5) yields

$$
\frac{\left|\lambda_{n}^{\prime}\left(x_{j}\right)\right|}{\lambda_{j}}\left|x-x_{j}\right| l_{j}^{2}(x) \leq C \frac{w\left(x_{j}\right)\left(1-x_{j}^{2}\right)^{1 / 2}}{n w(x)\left(1-x_{j}^{2}\right)} .
$$

Because $x_{j}$ is the nearest point to $x$ (according to the definition of $x_{j}$ ), we have $1-x^{2} \sim 1-x_{j}^{2}, w(x) \sim w\left(x_{j}\right)$. Hence,

$$
\frac{\left|\lambda_{n}^{\prime}\left(x_{j}\right)\right|}{\lambda_{j}}\left|x-x_{j}\right| l_{j}^{2}(x) \leq C \frac{\left(1-x_{j}^{2}\right)^{-1 / 2}}{n} \leq C .
$$


By using (2.14) and (2.4), we get

$$
\begin{aligned}
S & :=\sum_{k \neq j} \frac{\left|\lambda_{n}^{\prime}\left(x_{k}\right)\right|}{\lambda_{k}}\left|x-x_{k}\right| l_{k}^{2}(x) \leq C \sum_{k \neq j} \frac{1}{1-x_{k}^{2}}\left|x-x_{k}\right| l_{k}^{2}(x) \\
& \leq C \sum_{k \neq j} \frac{w\left(x_{k}\right)\left(1-x_{k}^{2}\right)^{1 / 2}}{n^{2}\left|x-x_{k}\right| w(x)\left(1-x^{2}\right)^{1 / 2}} .
\end{aligned}
$$

Further, we use (2.1) and (2.3) to obtain

$$
\begin{aligned}
S & \leq C \sum_{k \neq j} \frac{\left(1-x_{k}\right)^{\alpha+1 / 2}\left(1+x_{k}\right)^{\beta+1 / 2}}{n^{2}\left|x-x_{k}\right|\left(1-x_{j}\right)^{\alpha+1 / 2}\left(1+x_{j}\right)^{\beta+1 / 2}} \\
& \leq C \sum_{k \neq j} \frac{(k / n)^{2 \alpha+1}((n+1-k) / n)^{2 \beta+1}}{|k-j| \min \{k+j, 2 n+2-k-j\}(j / n)^{2 \alpha+1}} \\
& \leq C \frac{1}{n^{2 \beta+1} j^{2 \alpha+1}} \sum_{k \neq j} \frac{k^{2 \alpha+1}(n+1-k)^{2 \beta+1}}{|k-j| \min \{k+j, 2 n+2-k-j\}} .
\end{aligned}
$$

For $j \leq n / 2$, we have $k+j \leq 3 n / 2$, and hence

$$
2 n+2-k-j \geq 2 n+2-n-\frac{n}{2} \geq \frac{n}{2} \geq \frac{1}{3}(k+j),
$$

which implies that

$$
\min \{k+j, 2 n+2-k-j\} \geq \frac{1}{3}(k+j) .
$$

Thus,

$$
\begin{aligned}
& S \leq C\left[\frac{1}{n^{2 \beta+1} j^{2 \alpha+1}} \sum_{\substack{k \leq 3 n / 4 \\
k \neq j}} \frac{n^{2 \beta+1} k^{2 \alpha+1}}{\left|k^{2}-j^{2}\right|}\right.\left.+\frac{1}{n^{2 \beta+1} j^{2 \alpha+1}} \sum_{k>3 n / 4} \frac{n^{2 \alpha+1}(n+1-k)^{2 \beta+1}}{\left|k^{2}-j^{2}\right|}\right] \\
& \leq C\left[\frac{1}{j^{2 \alpha+1}} \sum_{\substack{k \leq 3 n / 4 \\
k \neq j}} \frac{k^{2 \alpha+1}}{\left|k^{2}-j^{2}\right|}+\frac{n^{2 \alpha}}{j^{2 \alpha+1}}\right] .
\end{aligned}
$$

Put

$$
\begin{array}{ll}
k_{1}=\left\{k: k \leq \frac{j}{2}\right\}, & k_{2}=\left\{k: \frac{j}{2}<k \leq \frac{3 j}{2}, k \neq j\right\}, \\
k_{3}=\left\{k: \frac{3 j}{2}<k \leq \frac{3 n}{4}\right\}, & S_{i}=\sum_{k \in k_{i}} \frac{k^{2 \alpha+1}}{\left|k^{2}-j^{2}\right|}, \quad i=1,2,3 .
\end{array}
$$


We estimate $S_{i}$ individually as follows:

$$
\begin{aligned}
& S_{1}=\sum_{k \in k_{1}} \frac{k^{2 \alpha+1}}{\left|k^{2}-j^{2}\right|} \leq C \sum_{k \in k_{1}} \frac{k^{2 \alpha+1}}{j^{2}} \leq C j^{2 \alpha}, \\
& S_{2}=\sum_{k \in k_{2}} \frac{k^{2 \alpha+1}}{\left|k^{2}-j^{2}\right|} \leq C \sum_{k \in k_{2}} \frac{j^{2 \alpha+1}}{j|k-j|}=C \sum_{k \in k_{2}} \frac{j^{2 \alpha}}{|k-j|} \leq C j^{2 \alpha} \ln j .
\end{aligned}
$$

Since $k \in k_{3}$ implies $k-j>k / 3$, one has

$$
\begin{aligned}
S_{3} & =\sum_{k \in k_{3}} \frac{k^{2 \alpha+1}}{\left|k^{2}-j^{2}\right|} \leq C \sum_{k \in k_{3}} \frac{k^{2 \alpha+1}}{|k+j| k} \leq C j^{-1} \sum_{k \in k_{3}} k^{2 \alpha} \\
& = \begin{cases}C j^{-1}, & \alpha<-\frac{1}{2}, \\
C j^{-1} \ln n, & \alpha=-\frac{1}{2}, \\
C j^{-1} n^{2 \alpha+1}, & \alpha>-\frac{1}{2},\end{cases}
\end{aligned}
$$

that is,

$$
S_{3} \leq \begin{cases}C j^{-1}, & \alpha<-\frac{1}{2}, \\ C j^{-1} n^{2 \alpha+1} \ln n, & \alpha \geq-\frac{1}{2} .\end{cases}
$$

Thus we have, for $\alpha<-1 / 2$,

$$
S \leq C j^{-2 \alpha-2}=C \frac{1}{n^{2 \alpha+2}}\left(\frac{n}{j}\right)^{2 \alpha+2} \leq C \frac{1}{n^{2 \alpha+2} w(x)\left(1-x^{2}\right)}
$$

and, for $\alpha \geq-1 / 2$,

$$
S \leq C \frac{\ln n}{n}\left(\frac{n}{j}\right)^{2 \alpha+2} \leq C \frac{\ln n}{n w(x)\left(1-x^{2}\right)} .
$$

It follows that, for $0 \leq x \leq 1-\sigma n^{-2}$,

$$
S \leq C\left[\frac{1}{n^{2 \alpha+2}}+\frac{\ln n}{n}\right] \frac{1}{w(x)\left(1-x^{2}\right)} .
$$

The proof for $-1+\sigma n^{-2} \leq x \leq 0$ is similar. Finally, we get

$$
S \leq C\left[\frac{1}{n^{2 \beta+2}}+\frac{\ln n}{n}\right] \frac{1}{w(x)\left(1-x^{2}\right)} .
$$


Hence,

$$
\begin{aligned}
& \sum_{k=1}^{n} \frac{\left|\lambda_{n}^{\prime}\left(x_{k}\right)\right|}{\lambda_{k}}\left|x-x_{k}\right| l_{k}^{2}(x) \\
& \quad \leq \text { const }\left[\frac{1}{n^{2 \alpha+2}}+\frac{1}{n^{2 \beta+2}}+\frac{\ln n}{n}\right] \frac{1}{w(x)\left(1-x^{2}\right)}
\end{aligned}
$$

This proves (2.12).

The proof of (2.13) is similar to that of (2.12) with a slight modification for the estimate of $S_{3}\left(0 \leq x \leq 1-\sigma n^{-2}\right)$. A direct computation leads to

$$
\begin{aligned}
S_{3} & =\sum_{k \in k_{3}} \frac{k^{2 \alpha+1}}{\left|k^{2}-j^{2}\right|} \leq C \sum_{k \in k_{3}} \frac{k^{2 \alpha+1}}{|k+j| k} \leq C \sum_{k \in k_{3}} \frac{k^{2 \alpha+1}}{k^{2}} \\
& =C \sum_{k \in k_{3}} k^{2 \alpha-1} \leq \begin{cases}C, & \alpha<0, \\
C n^{2 \alpha} \ln n, & \alpha \geq 0 .\end{cases}
\end{aligned}
$$

Thus, we obtain, for $\alpha<0$,

$$
S \leq C j^{-2 \alpha-1}=C \frac{1}{n^{2 \alpha+1}}\left(\frac{n}{j}\right)^{2 \alpha+1} \leq C \frac{1}{n^{2 \alpha+1} w(x)\left(1-x^{2}\right)^{1 / 2}},
$$

and, for $\alpha \geq 0$,

$$
S \leq C \frac{\ln n}{n}\left(\frac{n}{j}\right)^{2 \alpha+1} \leq C \frac{\ln n}{n w(x)\left(1-x^{2}\right)^{1 / 2}} .
$$

Combining these two cases gives

$$
S \leq \text { const }\left[\frac{1}{n^{2 \alpha+1}}+\frac{\ln n}{n}\right] \frac{1}{w(x)\left(1-x^{2}\right)^{1 / 2}} .
$$

The proof for the case $-1+\sigma n^{-2} \leq x \leq 0$ is also similar. Finally, we conclude

$$
\begin{aligned}
& \sum_{k=1}^{n} \frac{\left|\lambda_{n}^{\prime}\left(x_{k}\right)\right|}{\lambda_{k}}\left|x-x_{k}\right| l_{k}^{2}(x) \\
& \quad \leq \text { const }\left[\frac{1}{n^{2 \alpha+1}}+\frac{1}{n^{2 \beta+1}}+\frac{\ln n}{n}\right] \frac{1}{w(x)\left(1-x^{2}\right)^{1 / 2}} .
\end{aligned}
$$

This proves (2.13).

3. Main results. In this section, we present two results about weighted $L^{p}$ convergence for Grünwald interpolation with the special case in which we obtain a sufficient and necessary condition. 
THEOREM 3.1. Let $w$ be a weight function. Then

$$
\lim _{n \rightarrow \infty}\left\|G_{n}(f, x)-f\right\|_{w, 1}=0, \quad \text { for every } f \in C[-1,1]
$$

if and only if

$$
\lim _{n \rightarrow \infty}\left\|\sum_{k=1}^{n} l_{k}^{2}(x)-1\right\|_{w, 1}=0
$$

Proof. (1) It is easy to see that

$$
\left\|G_{n}(1, x)-1\right\|_{w, 1} \longrightarrow 0 \Longleftrightarrow\left\|\sum_{k=1}^{n} l_{k}^{2}(x)-1\right\|_{w, 1} \longrightarrow 0, \quad(n \longrightarrow \infty) .
$$

(2) For $\phi_{x}(t)=(x-t)^{2}$, we have

$$
G_{n}\left(\phi_{x}(x), x\right)=\sum_{k=1}^{n}\left(x-x_{k}\right)^{2} l_{k}^{2}(x)
$$

By Hermite interpolation, we know that

$$
H_{n}\left(\phi_{x}(x), x\right)=2 \sum_{k=1}^{n}\left(x-x_{k}\right)^{2} l_{k}^{2}(x)
$$

Using Lemma 2.2, we find that (3.5) converges to 0. Thus (3.4) converges to 0. According to Lemma 2.1, (3.1) holds if and only if

$$
\lim _{n \rightarrow \infty}\left\|G_{n}(x, f)-f\right\|_{w, 1}=\lim _{n \rightarrow \infty}\left\|\sum_{k=1}^{n} l_{k}^{2}(x)-1\right\|_{w, 1}=0
$$

THEOREM 3.2. Let $f \in C[-1,1]$. Let $w \in G \mathrm{~J}$ with parameters $\alpha, \beta$, and $u$ be Jacobi weight function with parameters $a, b$, where $a, b, \alpha, \beta>-1$. If

$$
\begin{aligned}
& p \alpha-a-1<\min \{0, p \beta\}, \\
& p \beta-b-1<\min \{0, p \alpha\},
\end{aligned}
$$

then

$$
\lim _{n \rightarrow \infty}\left\|G_{n}(w, f, x)-f(x)\right\|_{u, p}=0
$$


Conversely, if (3.8) holds, then

$$
\begin{aligned}
& p \alpha-a-1<0, \\
& p \beta-b-1<0 .
\end{aligned}
$$

Proof. Assume that (3.7) holds.

Let $f$ be a polynomial of degree $<2 n$. By definitions, we have

$$
\begin{aligned}
f(x)-G_{n}(w, f, x)= & f(x)-H_{n}(w, f, x) \\
& +\sum_{k=1}^{n} f\left(x_{k}\right) \frac{\lambda_{n}^{\prime}\left(x_{k}\right)}{\lambda_{k}}\left(x-x_{k}\right) l_{k}^{2}(x) .
\end{aligned}
$$

Then

$$
\begin{aligned}
I & :=\left\|G_{n}(w, f, x)-f(x)\right\|_{u, p}^{p}=\int_{-1}^{1}\left|f(x)-G_{n}(w, f, x)\right|^{p} u(x) d x \\
& =\int_{-1}^{1}\left|f(x)-H_{n}(w, f, x)+\sum_{k=1}^{n} f\left(x_{k}\right) \frac{\lambda_{n}^{\prime}\left(x_{k}\right)}{\lambda_{k}}\left(x-x_{k}\right) l_{k}^{2}(x)\right|^{p} u(x) d x .
\end{aligned}
$$

It follows from (2.8) and (2.10) that

$$
\begin{aligned}
I \leq & C \int_{-1}^{1}\left|f(x)-H_{n}(w, f, x)\right|^{p} u(x) d x \\
& +C \int_{-1}^{1}\left|\sum_{k=1}^{n} f\left(x_{k}\right) \frac{\lambda_{n}^{\prime}\left(x_{k}\right)}{\lambda_{k}}\left(x-x_{k}\right) l_{k}^{2}(x)\right|^{p} u(x) d x, \\
\leq & C\left\|f-H_{n}(f)\right\|_{u, p}^{p} \\
& +C\|f\|_{\infty}^{p} \int_{-1+\sigma n^{-2}}^{1-\sigma n^{-2}}\left[\sum_{k=1}^{n}\left|\frac{\lambda_{n}^{\prime}\left(x_{k}\right)}{\lambda_{k}}\left(x-x_{k}\right) l_{k}^{2}(x)\right|\right]^{p} u(x) d x \\
& :=I_{1}+I_{2} .
\end{aligned}
$$

We next estimate $I_{1}$ and $I_{2}$.

By Lemma 2.4, we know that $w^{-1} \in L_{u}^{p}$ implies $I_{1} \rightarrow 0(n \rightarrow \infty)$. Obviously, $w^{-1} \in L_{u}^{p}$ is equivalent to (3.9). According to Lemma 2.5 and (2.12), we get

$$
\begin{aligned}
I_{2} & =C \int_{-1+\sigma n^{-2}}^{1-\sigma n^{-2}}\left[\sum_{k=1}^{n} \frac{\left|\lambda_{n}^{\prime}\left(x_{k}\right)\right|}{\lambda_{k}}\left|x-x_{k}\right| l_{k}^{2}(x)\right]^{p} u(x) d x \\
& \leq C\left[\frac{1}{n^{2 \alpha+2}}+\frac{1}{n^{2 \beta+2}}+\frac{\ln n}{n}\right]^{p} \int_{-1+\sigma n^{-2}}^{1-\sigma n^{-2}}\left[\frac{1}{w(x)\left(1-x^{2}\right)}\right]^{p} u(x) d x .
\end{aligned}
$$


It can be shown that

$$
\begin{aligned}
I_{3} & :=\int_{-1+\sigma n^{-2}}^{1-\sigma n^{-2}}\left[\frac{1}{w(x)\left(1-x^{2}\right)}\right]^{p} u(x) d x \\
& \leq C\left[1+n^{2 p \alpha-2 a+2 p-2}+n^{2 p \beta-2 b+2 p-2}\right] .
\end{aligned}
$$

Then,

$$
\begin{aligned}
I_{2} \leq C & {\left[\frac{1}{n^{2 \alpha+2}}+\frac{1}{n^{2 \beta+2}}+\frac{\ln n}{n}\right]^{p} \int_{-1+\sigma n^{-2}}^{1-\sigma n^{-2}}\left[\frac{1}{w(x)\left(1-x^{2}\right)}\right]^{p} u(x) d x } \\
\leq C & {\left[n^{-2 p(\alpha+1)}+n^{-2 p(\beta+1)}+\left[\frac{\ln n}{n}\right]^{p}+n^{-2(a+1)}+n^{-2(b+1)}\right.} \\
& +n^{2 p \alpha-2 a+p-2}(\ln n)^{p}+n^{2 p \beta-2 b-2 p \alpha-2} \\
& \left.+n^{2 p \alpha-2 a-2-2 p \beta}+n^{2 p \beta-2 b+p-2}(\ln n)^{p}\right] .
\end{aligned}
$$

Recall that $\alpha+1>0, \beta+1>0, a+1>0$, and $b+1>0$. Therefore, when

$$
\begin{aligned}
& p \alpha-a-1<\min \left\{-\frac{p}{2}, p \beta\right\}, \\
& p \beta-b-1<\min \left\{-\frac{p}{2}, p \alpha\right\}
\end{aligned}
$$

we have $I_{2} \rightarrow 0$ (as $n \rightarrow \infty$ ). Obviously, (3.16) implies (3.9).

On the other hand, for $I_{2}$, if we use (2.13) in Lemma 2.5, we can get

$$
I_{2} \leq C\left[\frac{1}{n^{2 p \alpha+p}}+\frac{1}{n^{2 p \beta+p}}+\frac{(\ln n)^{p}}{n^{p}}\right] \int_{-1+\sigma n^{-2}}^{1-\sigma n^{-2}}\left[\frac{1}{w(x)\left(1-x^{2}\right)^{1 / 2}}\right]^{p} u(x) d x
$$

Again we need to estimate the integral

$$
\begin{aligned}
I_{4} & :=\int_{-1+\sigma n^{-2}}^{1-\sigma n^{-2}}\left[\frac{1}{w(x)\left(1-x^{2}\right)^{1 / 2}}\right]^{p} u(x) d x \\
& \leq C\left[1+n^{2 p \beta-2 b-2+p}+n^{2 p \alpha-2 a-2+p}\right] .
\end{aligned}
$$


Thus

$$
\begin{aligned}
I_{2} \leq C & {\left[\frac{1}{n^{2 p \alpha+p}}+\frac{1}{n^{2 p \beta+p}}+\frac{(\ln n)^{p}}{n^{p}}\right] \int_{-1+\sigma n^{-2}}^{1-\sigma n^{-2}}\left[\frac{1}{w(x)\left(1-x^{2}\right)^{1 / 2}}\right]^{p} u(x) d x } \\
\leq C & {\left[n^{-p(2 \alpha+1)}+n^{-p(2 \beta+1)}+\left(\frac{\ln n}{n}\right)^{p}+n^{-2 a-2}+n^{-2 b-2}+n^{2 p \beta-2 b-2 p \alpha-2}\right.} \\
& \left.+n^{2 p \alpha-2 a-2-2 p \beta}+n^{2 p \beta-2 b-2}(\ln n)^{p}+n^{2 p \alpha-2 a-2}(\ln n)^{p}\right] .
\end{aligned}
$$

Similarly when

$$
\begin{gathered}
\alpha, \beta>-\frac{1}{2}, \\
p \alpha-a-1<\min \{0, p \beta\}, \\
p \beta-b-1<\min \{0, p \alpha\},
\end{gathered}
$$

we have $I_{2} \rightarrow 0($ as $n \rightarrow \infty)$. We have proved that when

$$
\begin{aligned}
& p \alpha-a-1<\min \{0, p \beta\}, \quad p \beta-b-1<\min \{0, p \alpha\}, \quad \text { if } \alpha, \beta>-\frac{1}{2}, \\
& p \alpha-a-1<\min \left\{-\frac{p}{2}, p \beta\right\}, \quad p \beta-b-1<\min \left\{-\frac{p}{2}, p \alpha\right\}, \quad \text { otherwise, }
\end{aligned}
$$

(3.8) holds.

It remains to prove that (3.21) is equivalent to (3.7). If (3.7) is true, we only need to prove that when $\alpha \leq-1 / 2$ or $\beta \leq-1 / 2$, (3.21) holds. Let $\alpha \leq-1 / 2$. Then,

$$
p \alpha-a-1<p \alpha \leq-\frac{p}{2}
$$

This inequality with $p \alpha-a-1<p \beta$ in (3.7) gives

$$
p \alpha-a-1<\min \left\{-\frac{p}{2}, p \beta\right\}
$$

On the other hand, from $p \beta-b-1<\min \{0, p \alpha\}$ in (3.7), we obtain that

$$
p \beta-b-1<\min \{0, p \alpha\}=p \alpha=\min \left\{-\frac{p}{2}, p \alpha\right\} .
$$

Conversely, if (3.21) is true, it is obvious that (3.7) is true because

$$
\begin{aligned}
& p \alpha-a-1<\min \left\{-\frac{p}{2}, p \beta\right\} \leq \min \{0, p \beta\}, \\
& p \beta-b-1<\min \left\{-\frac{p}{2}, p \alpha\right\} \leq \min \{0, p \alpha\} .
\end{aligned}
$$

Now we can show the second part of the theorem.

Assume that (3.8) is true. To this end, we put $f_{0}:=1$ and $f_{1}:=x$. Then, by 
[8, Theorem 3, page 51], we have

$$
\begin{aligned}
\left|f_{1}-H_{n}\left(f_{1}\right)\right| & =\left|\sum_{k=1}^{n}\left(x-x_{k}\right) l_{k}^{2}(x)\right| \\
& =\left|x G_{n}\left(f_{0}\right)-G_{n}\left(f_{1}\right)\right| \\
& =\left|x\left[G_{n}\left(f_{0}\right)-f_{0}\right]+\left[f_{1}-g_{n}\left(f_{1}\right)\right]\right| \\
& \leq\left|G_{n}\left(f_{0}\right)-f_{0}\right|+\left|G_{n}\left(f_{1}\right)-f_{1}\right| .
\end{aligned}
$$

By (2.8), we have

$$
\begin{aligned}
& \left\|H_{n}\left(f_{1}\right)-f_{1}\right\|_{u, p}^{p} \\
& \quad \leq 2^{p}\left(\left\|G_{n}\left(f_{0}\right)-f_{0}\right\|_{u, p}^{p}+\left\|G_{n}\left(f_{1}\right)-f_{1}\right\|_{u, p}^{p}\right) \rightarrow 0, \quad 0<p<\infty .
\end{aligned}
$$

By using [8, Theorem 3, page 51], we prove $w^{-1} \in L_{u}^{p}$. This is equivalent to saying that (3.9) is true.

This completes the proof.

As an immediate consequence of Theorem 3.2, we state the following corollary.

COROLlary 3.3. Let $\alpha, \beta \geq 0$. Then (3.8) is equivalent to condition (3.9).

ACKNOWLEDGments. The author would like to thank Professor Yingguang Shi for his guidance. This work was supported by the National Natural Foundation of China.

\section{REFERENCES}

[1] E. W. Cheney, Introduction to Approximation Theory, McGraw-Hill Book Company, New York, 1966.

[2] G. Freud, Orthogonal Polynomials, Pergamon Press, Oxford, 1971.

[3] G. Grünwald, On the theory of interpolation, Acta Math. 75 (1943), 219-245.

[4] W. Kitto and D. E. Wulbert, Korovkin approximations in $L_{p}$-spaces, Pacific J. Math. 63 (1976), no. 1, 153-167.

[5] G. Min, $L^{1}$-convergence of Grünwald interpolation operators, Adv. in Math. (Beijing) 18 (1989), no. 4, 485-490 (Chinese).

[6] _ On Grünwald's interpolation operator and its applications, J. Math. Res. Exposition 9 (1989), no. 3, 442-446 (Chinese).

[7] P. Nevai, Orthogonal polynomials, Mem. Amer. Math. Soc. 18 (1979), no. 213, $\mathrm{v}+185$.

[8] P. Nevai and P. Vértesi, Mean convergence of Hermite-Fejér interpolation, J. Math. Anal. Appl. 105 (1985), no. 1, 26-58.

[9] G. Szegö, Orthogonal Polynomials, revised ed., American Mathematical Society Colloquium Publications, vol. 23, American Mathematical Society, Rhode Island, 1959.

Zhixiong Chen: Department of Mathematics, University of Connecticut, Storrs, CT 06269, USA

E-mail address: zchen@math.uconn.edu 


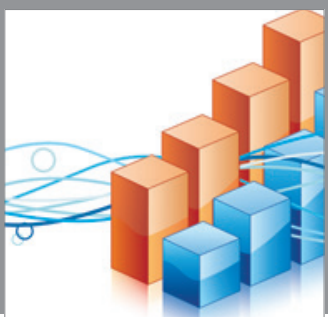

Advances in

Operations Research

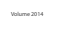

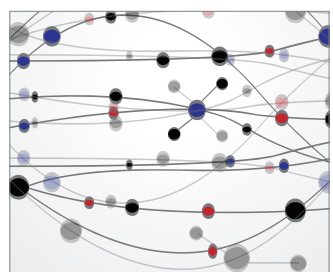

\section{The Scientific} World Journal
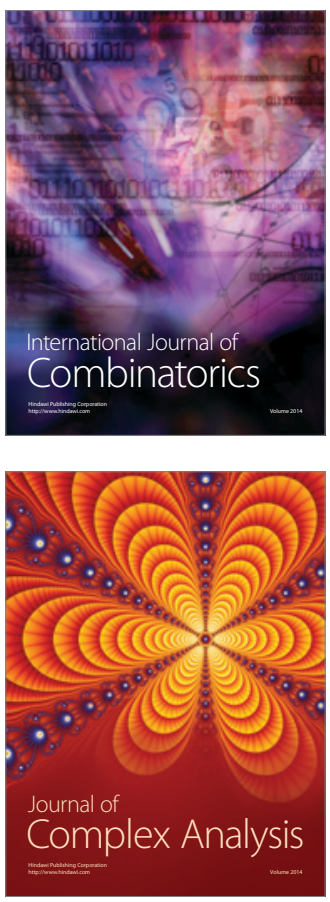

International Journal of

Mathematics and

Mathematical

Sciences
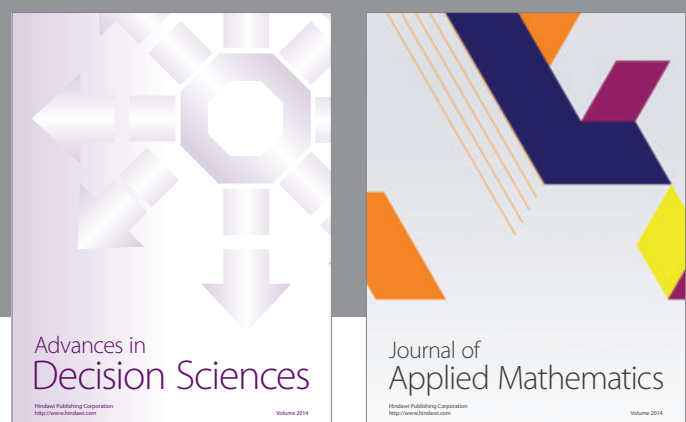

Journal of

Applied Mathematics
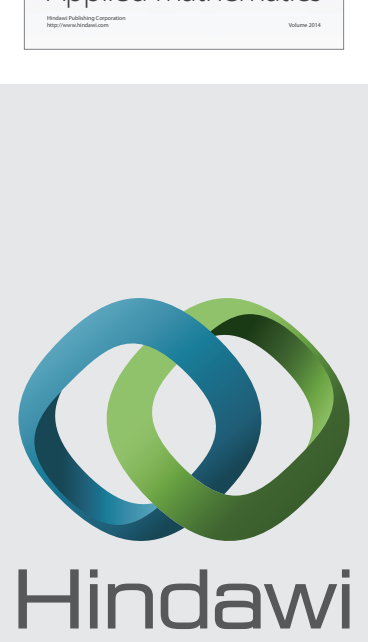

Submit your manuscripts at http://www.hindawi.com
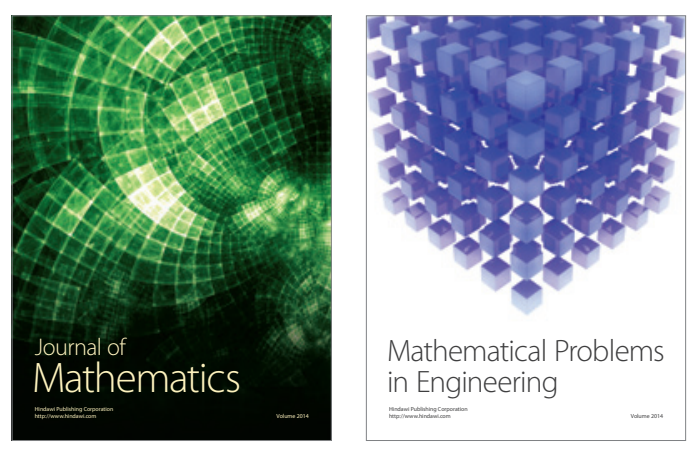

Mathematical Problems in Engineering
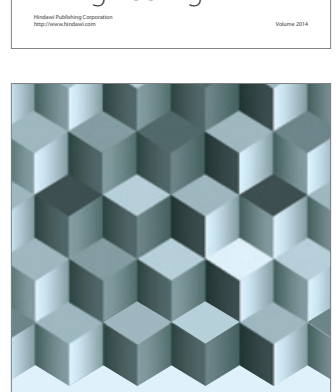

Journal of

Function Spaces
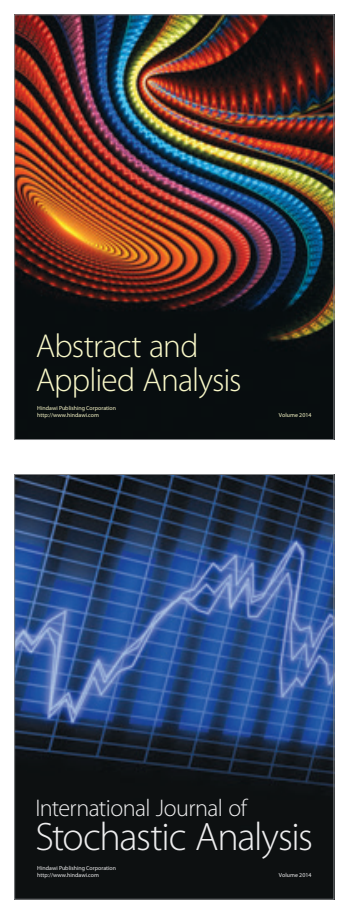

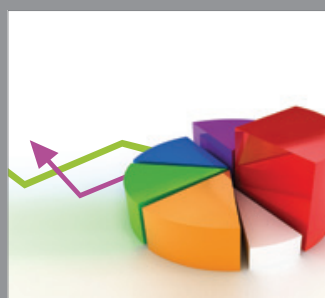

ournal of

Probability and Statistics

Promensencen
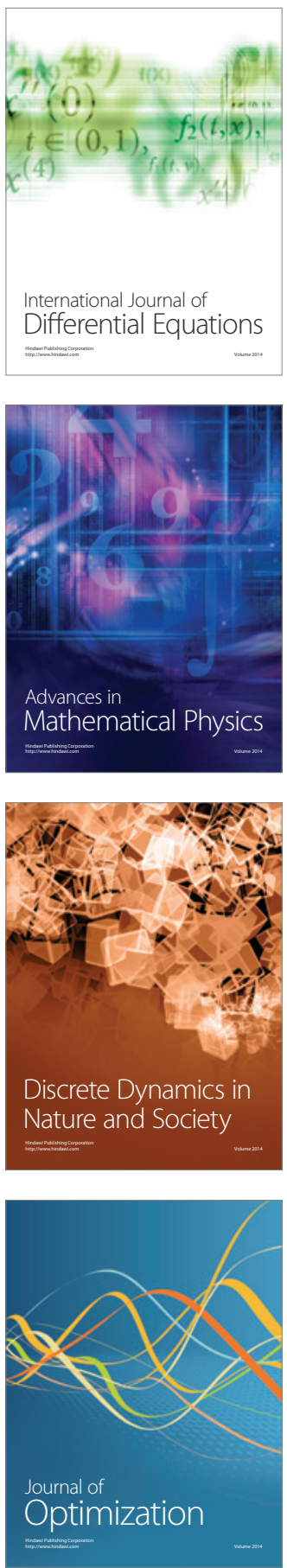